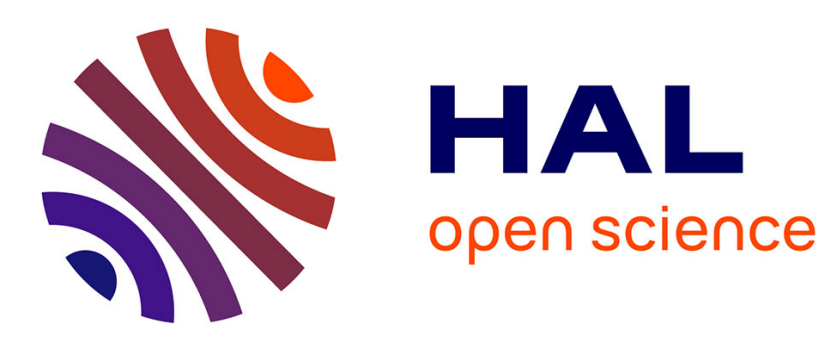

\title{
Aider l'étudiant infirmier à acquérir une posture réflexive
}

\author{
Patricia Jomas
}

\section{To cite this version:}

Patricia Jomas. Aider l'étudiant infirmier à acquérir une posture réflexive. Les Métiers de la petite enfance, 2018, 24, pp.12 - 14. 10.1016/j.melaen.2018.10.005 . hal-03487663

\section{HAL Id: hal-03487663 \\ https://hal.science/hal-03487663}

Submitted on 20 Dec 2021

HAL is a multi-disciplinary open access archive for the deposit and dissemination of scientific research documents, whether they are published or not. The documents may come from teaching and research institutions in France or abroad, or from public or private research centers.
L'archive ouverte pluridisciplinaire HAL, est destinée au dépôt et à la diffusion de documents scientifiques de niveau recherche, publiés ou non, émanant des établissements d'enseignement et de recherche français ou étrangers, des laboratoires publics ou privés.

\section{다)(1) $(5$}

Distributed under a Creative Commons Attribution - NonCommercial| 4.0 International 


\title{
Dochead dossier
}

\section{Sous-dochead Accueillir un stagiaire}

\author{
professionnalisation
}

\section{Aider l'étudiant infirmier à acquérir une posture réflexive}

\author{
Patricia Jomas \\ Cadre de santé formateur \\ Institut de formation en soins infirmiers Bicêtre/Paul Brousse, AP-HP, 78, rue du Général-Leclerc, \\ 94270 Le Kremlin-Bicêtre, France \\ Adresse e-mail : patricia.jomas@aphp.fr (P. Jomas).
}

\section{Résumé}

La posture réflexive est inscrite dans la formation infirmière depuis 2009 et est incluse dans celle des enseignants. Différentes modalités pédagogiques permettent d'accompagner l'apprenant vers une finalité : la réflexion comme construction de la professionnalisation. Pour cela, l'étudiant s'appuie sur l'expérience du formateur, qui l'initie à la méthodologie de l'analyse de situations, et sur celle du professionnel de terrain, qui questionne sa pratique.

(C) 2018

Mots clés - étudiant infirmier ; formation ; pédagogie ; pratique réflexive ; profession

Après la mise en place des textes relatifs à la formation d'aide-soignant [1] et d'auxiliaire de puériculture [2], la formation infirmière a évolué en 2009. Le référentiel de formation mis en place [3] est construit autour de l'acquisition de dix compétences qui sont fondamentales pour répondre aux besoins de santé des personnes, dans le cadre d'une pluriprofessionnalité.

\section{T1 Finalité de la formation}

Le référentiel de formation des infirmiers est pensé en termes de "construction". L'étudiant participe pleinement à l'élaboration du professionnel en devenir qu'il est, dès l'entrée en institut de formation. En effet, "il est amené à devenir un praticien autonome, responsable et réflexif » [3]. L'étudiant deviendra un professionnel capable de prendre les décisions adaptées aux situations rencontrées et de mettre en œuvre les actions nécessaires, seul ou en équipe, dans la limite de son rôle défini par le Code de la santé publique [4] ; sous-entendu, il sera capable d'analyser toute situation de santé au sens large.

Par ailleurs, "l'étudiant développe des ressources» [3]. Celles-ci concernent les connaissances théoriques et la méthodologie enseignées au cours des trois ans de formation, 
mais aussi la technique liée aux différents soins et les aptitudes relationnelles. "L'étudiant apprend à reconnaître ses émotions » afin d'interagir avec justesse dans ses relations avec l'autre. Il gagne en assurance, ce qui lui permet de se positionner tout en questionnant sa pratique. Enfin, «il développe une éthique professionnelle » qui lui permet de questionner la situation et de faire des choix éclairés.

Donald A. Schön [5,6], pédagogue influent dans le monde anglo-saxon, est à l'origine de travaux sur les stratégies d'apprentissage réflexives par la pratique. Selon lui, la pratique réflexive consiste à apprendre par l'action et dans l'action, d'où la nécessité d'acquérir des savoirs et de les mettre en œuvre puis de se former à l'analyse de sa pratique.

« Cette démarche conduit à :

- améliorer sa pratique, devenir plus efficace (aller plus vite, diminuer les coûts...),

- contrôler ses actions : observer comment se fait "réellement" le travail, analyser les écarts entre tâche prescrite et tâche réelle,

- valider sa pratique : poser un regard sur ses actions, les valider (qualité du service rendu),

- faire émerger de nouveaux savoirs, découvrir des savoirs à partir de la pratique,

- donner du sens à sa pratique, améliorer la confiance en soi, accéder à une reconnaissance professionnelle »[5,6].

\section{T1 Méthodes pédagogiques}

\section{TEG1 L'acquisition des dix compétences nécessaires à l'exercice de la profession} infirmière demande d'articuler l'acquisition de connaissances et de savoir-faire en lien avec des situations professionnelles. Les cours théoriques, toutes matières confondues, constituent la base sur laquelle s'appuyer pour construire les travaux dirigés. Les concepts en sciences infirmières et autres disciplines permettent à l'étudiant de travailler sur ses représentations. Dès le début de la formation, celui-ci est confronté à une remise en question de l'idéal du métier qu'il s'était construit antérieurement. Le premier stage conforte l'idée qu'il est incontournable de pratiquer régulièrement l'analyse de situations professionnelles.

TEG1 L'étude de ces situations permet de travailler trois paliers d'apprentissage :

- “comprendre", c'est-à-dire acquérir des connaissances et un savoir-faire indispensables à la compréhension des situations aussi bien en stage qu'à l'institut. Il s'agit de comprendre pour mesurer l'écart entre le prescrit et le réel, et pour donner du sens à sa pratique professionnelle ;

- “agir", l'étudiant utilise ses connaissances et sélectionne ses savoirs afin d'acquérir la capacité de mettre en œuvre des actions et de les évaluer ;

- “transférer”, qui nécessite pour l'étudiant de conceptualiser sa pratique afin d'acquérir la capacité à transposer ses acquis dans de futures situations pouvant devenir de plus en plus complexes.

L'un des points forts de l'apprentissage est constitué de l'analyse de situations, exercice à réaliser à chaque période de stage de cinq semaines, et la production du travail écrit de fin 
d'études, le mémoire, qui s'inscrit dans l'unité d'enseignement 5.6 du semestre 6 "Intégration des savoirs et posture professionnelle infirmière".

\section{T2 L'analyse de situations}

En ce qui concerne la situation à analyser, l'étudiant choisit une activité, un soin, une action qui se déroulent sur un temps défini. La situation peut concerner une technique de soins, un aspect relationnel, réglementaire... Le choix est laissé à l'étudiant, à condition que la situation soit en lien avec la spécificité du lieu de stage, avec ses objectifs personnels ou institutionnels ou avec les situations prévalentes rencontrées dans la structure. La situation doit être présentée au tuteur de stage qui en valide la pertinence et la faisabilité [7]. Un autre critère à prendre en compte est celui de l'implication de l'étudiant dans la situation. Ce dernier doit être acteur afin de réfléchir à sa propre pratique.

Une description précise est demandée afin d'optimiser l'identification des axes à travailler. Pour cela, le raisonnement quintilien (questionnement "Qui ? Quoi ? Où ? Quand? Comment? Combien ? Pourquoi ?") est utilisé. Il s'agit d'une méthode empirique de questionnement qui permet à l'étudiant d'apprendre à structurer sa pensée et ainsi organiser son analyse. La carte conceptuelle [8] est également un outil intéressant pour l'étudiant dont la méthode d'apprentissage est plutôt visuelle.

La finalité de l'exercice consiste à mesurer l'écart entre "le prescrit" et "le réel". Dans la situation vécue, qu'est-ce qui était attendu ? Les règles, les textes réglementaires, les protocoles, les procédures, les pratiques de soins, l'éthique sont des éléments de référence sur lesquels l'étudiant s'appuie pour évaluer le résultat obtenu. De multiples questions surgissent alors : qu'est-ce qui fait que le résultat obtenu est satisfaisant ou non ? Quelles sont mes ressources, intrinsèques et extrinsèques ? Sont-elles suffisantes ? Ai-je les connaissances ? Est-ce que je peux faire autrement ? Quel est l'élément déterminant dans la situation ?...

Par expérience, l'étudiant est tenté d'analyser les situations jugées "négatives", pour lesquelles il a le sentiment de ne pas avoir atteint son objectif. Il est important de l'aider à réfléchir aux situations jugées "positives" afin d'être capable d'identifier les éléments transférables à de nouvelles situations.

\section{T2 Le mémoire}

Pour la réalisation du mémoire, en semestre 6 de la formation, l'étudiant choisit une situation rencontrée durant sa formation. La plupart du temps, son choix s'oriente vers une situation qui l'a interpellé, ou qui peut être difficile à accepter pour lui. La motivation à travailler une situation dite "sociologique-anthropologique" est fréquente. Une situation vécue douloureusement, ou proche d'un vécu personnel, peut l'aider face à son besoin de comprendre, mais peut aussi diminuer son objectivité et sa capacité d'analyse. C'est pourquoi la validation du choix de la situation incombe au formateur/directeur de mémoire. 


\section{T1 Place du formateur et des professionnels}

Pour devenir un praticien réflexif, l'étudiant a besoin d'un accompagnement. Se questionner sur sa pratique, réfléchir à ses choix et ses hésitations, douter, remettre en question ses acquis ne sont pas des évidences pour tous les étudiants. Il s'agit d'un mode de pensée que certains possèdent déjà, avec une certaine curiosité, une envie de comprendre, de connaître davantage, de donner du sens à leurs actes. Pour d'autres, le chemin est plus long, d'où la nécessité pour le formateur d'accepter que l'étudiant n'adhère pas immédiatement à la méthodologie, ne comprenne pas l'intérêt de l'exercice et, parfois même, pense ne pas avoir besoin de s'initier à la pratique réflexive. Il s'agit alors de lui faire comprendre que la résolution de problème, seule, ne suffit pas. Patience et tolérance sont nécessaires pour celui qui accompagne l'étudiant dans ce processus. Il faut parfois accepter que celui-ci progresse peu, tout en ayant l'espoir que, devenu professionnel, il prenne conscience de ses acquis en formation et les mobilise afin de questionner sa pratique.

\section{Encadré flottant}

\section{La réflexivité pour un enrichissement mutuel}

TEG1 L'étudiant a parfois des difficultés à percevoir que l'infirmière déjà en poste a acquis les compétences lui permettant de questionner et d'analyser sa pratique tout en la réajustant. D'où la nécessité de l'implication du professionnel dans cette démarche réflexive. Le référentiel de 2009 prévoit ainsi un accompagnement par une infirmière qui connaît la finalité de cette formation, qui a compris que l'étudiant a besoin de questionner les pratiques et, bien évidemment, qui est capable d'accepter la confrontation d'idées.

TEG1 Cette volonté du référentiel de placer l'étudiant en tant qu'acteur et personne réflexive implique que les professionnels de la formation et ceux du terrain acceptent la remise en question. Comme dit précédemment, la théorie s'accompagne de travaux dirigés où le formateur illustre les connaissances par des situations cliniques. Ces temps permettent de décontextualiser la problématique pour mieux la travailler et la comprendre. L'étudiant doit s'autoriser à confronter ses connaissances à celles du formateur. L'attitude réflexive implique chez ce dernier la capacité à dire, parfois, qu'il ne sait pas, mais tout comme l'étudiant, il est en capacité de rechercher l'information qui lui permet de comprendre: la posture de formateur implique donc une certaine humilité. Ainsi, le formateur peut repenser sa façon d'encadrer l'étudiant, en lui demandant ce qu'il sait, ce qu'il a compris et pourquoi il fait de la sorte. Réduire le décalage entre ce que l'étudiant apprend à l'institut de formation et ce qu'il observe en stage est une finalité en partie accessible grâce à la réflexivité.

\section{T1 Conclusion}

L'acquisition d'une posture réflexive est une exigence de la formation infirmière qui doit permettre à l'étudiant de comprendre les liens entre les savoirs et leur mise en œuvre. Elle est 
une façon d'intégrer les connaissances théoriques à la construction de la compétence. Le référentiel de formation précise qu'il faut «revenir sur les acquis, les processus et les stratégies utilisées pour en dégager les principes transposables » [3]. Pour accompagner l'étudiant, le formateur s'appuie sur des modalités pédagogiques qui permettent à celui-ci de construire des savoirs. Il l'aide à développer son sens de l'observation, à rechercher des données scientifiques et à travailler à partir de sa propre expérience. "La réflexivité est une compétence clé qui doit être développée en formation initiale» [9]. Ce processus se poursuit une fois la formation achevée, grâce aux groupes d'analyse de pratiques qui constituent «des espaces de questionnement pour les professionnels » [10].

\section{Déclaration de liens d'intérêts}

L'auteur déclare ne pas avoir de liens d'intérêts.

\section{Références}

[1] Arrêté du 22 octobre 2005 relatif à la formation conduisant au diplôme d'État d'aidesoignant. www.legifrance.gouv.fr/affichTexte.do?cidTexte=JORFTEXT000000449527

[2] Arrêté du 16 janvier 2006 relatif à la formation conduisant au diplôme d'État d'auxiliaire de puériculture. www.legifrance.gouv.fr/affichTexte.do?cidTexte=JORFTEXT000000457507 [3] Arrêté du 31 juillet 2009 relatif au diplôme d'État d'infirmier. www.legifrance.gouv.fr/affichTexte.do?cidTexte=JORFTEXT000020961044

[4] Article R.4311-1 et suivants du Code de la santé publique. www.legifrance.gouv.fr/affichCode.do?idSectionTA=LEGISCTA000006190610\&cidTexte= LEGITEXT000006072665

[5] Schön DA. Le praticien réflexif. À la recherche du savoir caché dans l'agir professionnel. coll. Formation des maîtres. Montréal (Canada): Les Éditions Logiques; 1994.

[6] Vidalenc I, Malric M. Quels outils pour une démarche réflexive dans l'activité de recherche ? Interrogations. 2013;16.

[7] Agence régionale de santé d'Île-de-France. Évaluation des acquis de stage dans la formation initiale des infirmiers. www.chsf.fr/wp-content/uploads/2017/08/IFSI-Formationcontinue-Tutorat-Guide-\%C3\%A9val-acquis-ARS-IDF.pdf

[8] Bachelet R. Cartes conceptuelles et mind mapping. Cours École centrale de Lille. http://rb.eclille.fr/l/CarteConceptuelle/cours-cartes_conceptuelles.pdf

[9] Bouissou C, Brau-Antony S. Réflexivité et pratiques de formation. Regards critiques.

Carrefours de l'éducation. 2005;20(2):111-22.

[10] Zaoui E. Les groupes d'analyse des pratiques cliniques, une opportunité pour l'encadrement infirmier de renforcer le management des soins. Recherche en soins infirmiers. 2008;93:32-8.

Jomas-Illus1.jpg

(C) lenets_tan/stock-adobe.com

La pratique réflexive consiste à apprendre par l'action et dans l'action. 


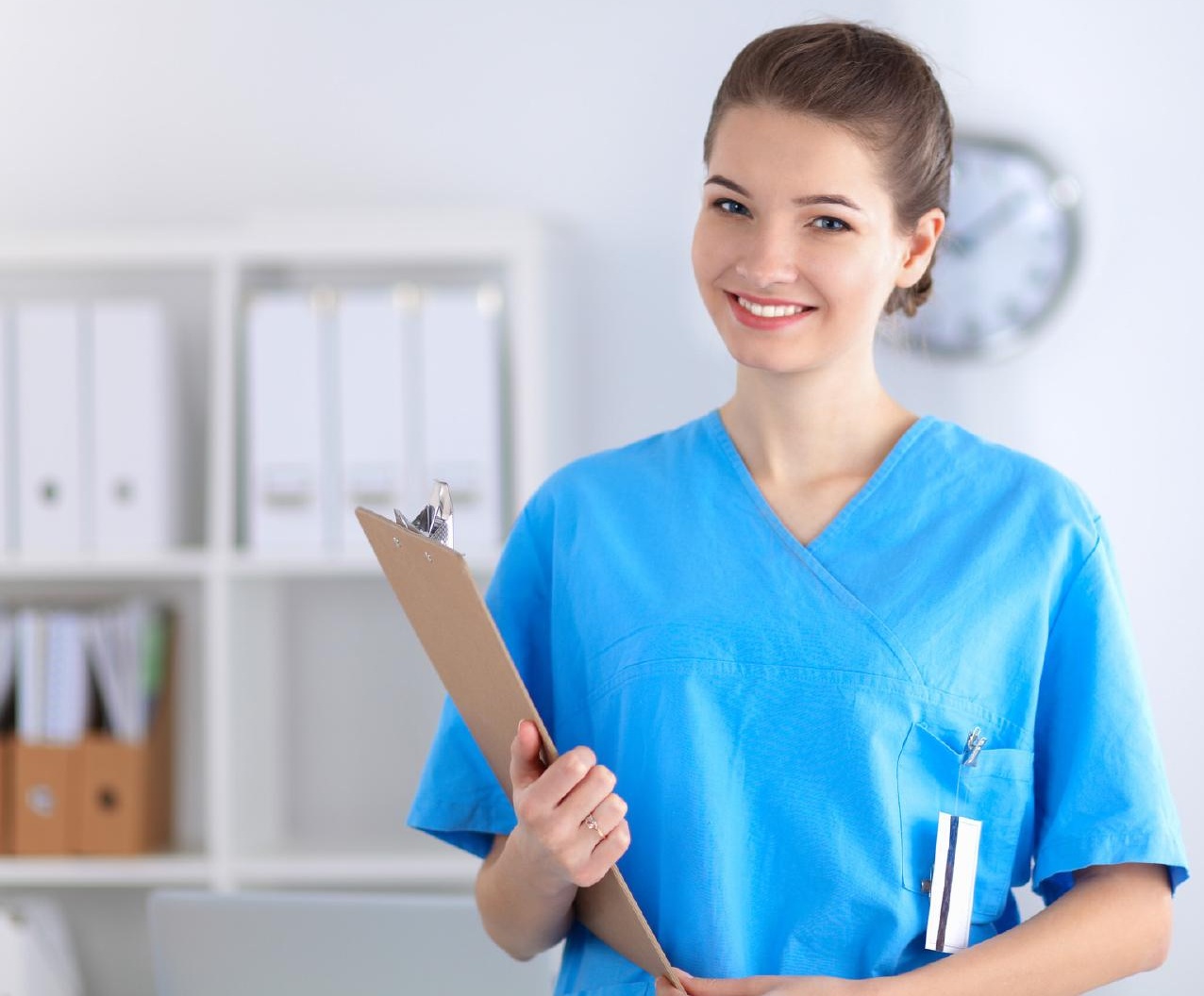

